\title{
Expert Panel Assessment of 57 Monocultivar Olive Oils Produced from the Tuscan Germplasm
}

\author{
C. Cantini ${ }^{1, *}$, G. Sani ${ }^{1}$, F. Gasperi ${ }^{2}$, F. Biasioli ${ }^{2}$ and E. Aprea ${ }^{2}$
}

${ }^{1}$ IVALSA CNR Via Aurelia 49, 58022 Follonica, Italy

${ }^{2}$ IASMA Research and Innovation Centre, Fondazione Edmund Mach, Food Quality and Nutrition Department, Via E. Mach, 1, 38010, S. Michele a/A, Italy

\begin{abstract}
An expert's panel according to the official method of International Olive Oil Council analyzed the organoleptic profile of the oils produced during one single harvesting season from 57 cultivars of the Tuscan germoplasm. The oils were grouped in three different clusters depending on the presence of different organoleptic features. The identified clusters of the extra virgin olive oils were compared for maturity index of the fruits and for the phenols content and acidic profile. Since phenols content and acidic profile depend on the genetic matrix hence the cultivar played a fundamental role on the overall quality. The majority of the varieties produced oils with the attributes requested by the local Protected Origin while others induced sensations, at the moment, not considered in the disciplinary of production like tomato or herbs. The presence of peculiar organoleptic attributes like tomato, sweet pepper, camomile or others is not common within the olive oils and they are present only to few varieties. The list of possible positive descriptors for the extra virgin olive oils could be enlarged or better specified including the terms of melon and chestnut flour. We proposed also the olfactory sensation of mushroom as reminiscent of the typical and pleasant odour of fresh cut mushroom as a possible new negative attribute of the extra virgin olive oil induced by the presence of antrachnose (Colletotrichum gloesporioides; Glomerella cingulata) on the olive fruits.
\end{abstract}

Keywords: Olea europaea L., cultivar characterisation, expert panel, official COI test, organoleptic descriptors, defect.

\section{INTRODUCTION}

The marketing of extra virgin olive oil (EVOO) is increasingly directed towards the differentiation, characterization and protection of the origins. Only in Italy there are, to date, over 40 European Protected Denominations of Origin (PDO). The production of EVOO using a designation of origin include the organoleptic assessment by which, a group of trained tasters, grant that the product holds the established characteristic sensory profile. This is a combination of olfactory, gustatory tactile and kinaesthetic characters produced by a combination of genetic base and pedoclimatic conditions. In Tuscany the oils with protected geographic origin (PGI) or protected denomination of origin (PDO) can be produced by several different varieties mixed together or with a single variety. In the last few years, the production of oils from a single variety, defined monovarietal or monocultivar, has increased [1] since the sensory character is strongly affected by the genotype of origin. Producing monovarietal oil could be a marketing strategy to differentiate the product from the mass but could be also a method for the valorization and maintaining of the autochthonous germplasm suited for local climates or environmental conditions. All this considered, the establishment of the organoleptic characteristics of the EVOO that can be produced from a single cultivar

*Address correspondence to this author at the IVALSA CNR, via Aurelia 49, 58022, Follonica, Italy; Tel/Fax: 390566 52356;

E-mail: cantini@ivalsa.cnr.it is fundamental both for the oils under designation of origins that for those declared monovarietal moreover, the sensory properties of EVOO play an important role in the evaluation of the overall quality and in the preference among consumers. Organoleptic assessment is also important because, even though several investigations were carried out to find relationships between sensory perceptions and volatiles [2-5] or non-volatiles [2] compounds, the results of the chemical analysis are not able to describe all the sensations experienced during the tasting [6].

Although many studies have been conducted [6-8] and others are still in progress, a complete characterization of the oil that can be produced by each cultivar is yet to come. This is due to the great number of olive cultivars worldwide, only in Italy for instance it has been estimated the presence of at least 500 different cultivated genotypes. The knowledge of the characteristics of the monocultivar olive oils producible within a germplasm collection is fundamental: it can be useful to understand the biodiversity, select plants for breeding, increase quality, define typicality and even more, valorize the positive attributes of the products going toward the expectations of the consumers [9].

With this research we studied the variation in the organoleptic profile of 57 Tuscan monocultivar EVOOs, produced in one single season, in order to gain information, select cultivars susceptible of a wider cultivation on the base of their positive characteristics and to enhance the valorization of the product on the market. We wanted also evaluate if the de- 
scriptors used so far for the evaluation of the various Tuscan Protected Origin extra virgin olive oils could cover all the possible characteristic producible within the Tuscan olive germplasm.

\section{MATERIALS AND METHODS}

Plant materials. The National Research Council of Italy collected the olive germplasm within the Tuscany region with a large research program conducted for more than 15 years. The autochthonous cultivars that have been identified are at the moment 79 and they have been put in collection at the Santa Paolina experimental station in Follonica (4256'30’'N, 1046’19'”E).

Each cultivar has been morphologically [10] and genetically [11] characterized and is maintained in collection as a group of four cloned plants. A detailed description of the cultivars with complete and up to date morphological and molecular information is available in Italian at http://olivo.arsia.toscana.it/olivotoscano/. The olive orchard, is cultivated in dry farming under typical Mediterranean environmental conditions, using traditional management practices. In the autumn 2010, during a two-months period, all the cultivars which presented enough fruits to produce the necessary quantity of oil for analysis were harvested following their stage of ripening. Forty kilos of healthy fruits were harvested for each 4-plant groups and the maturity index (MI) proposed by Uceda and Frías [12] was calculated for the fruits. The index serves as a common marker for maturation level and is based on the color of the fruit. It includes the following eight classes: intense green (0), yellowish green (1), green with reddish spots (2), reddish brown (3), black with white flesh (4), black with $<50 \%$ purple flesh (5), black with $\geq 50 \%$ purple flesh (6) and black with $100 \%$ purple flesh (7). The fruits were hand harvested in the morning and processed in the same afternoon to avoid any possible alteration.

Oil production. The olives, after the harvesting, were immediately washed and then crushed by a two-phases Oliomio® continuous mill (Toscana Enologica Mori, Tavarnelle V.P., Italy). This machinery reproduces, at a small scale, the industrial system of oil extraction so that the resulting EVOOs were similar to those producible at a commercial large-scale. The mechanical extraction, conducted with a minimum addition of water during the transport to the centrifuge $(2 \mathrm{~L} / \mathrm{h})$ was carried out to obtain an optimal recovery of biophenols which are related with several organoleptic attributes. All the operational conditions possibly affecting the quality of the oils (temperature and time of malaxation, speed of the centrifuge, flux of water in the separator) were kept steady to lower the variability among oil samples produced along the season. All the operations in the milling facility were carried out by a single operator. The oils at the exit of the horizontal centrifuge were immediately filtered with a cotton laboratory filter, divided in $100 \mathrm{ml}$ sample bottles and analyzed within 15 days from the extraction.

Oil characterisation, Determination of acidity and peroxide values of the samples was performed by a FoodLab analyzer (CDR, Ginestra Fiorentina, Firenze, Italy; http://foodlab.cdr-mediared.it). Phenolic compounds were identified according to International Olive Oil Council (IOOC) COI/T.20/Doc. No. 29 method and quantitative re- sults were expressed in $\mathrm{mg} / \mathrm{kg}$ of tyrosol. The sensory analysis of the samples was performed by the expert panel of the chamber of commerce of Grosseto, recognized by Italian Ministry for Agriculture under the conditions described in the IOOC regulations (COI/T.20/Doc. No. 15/Rev. 1, 1996 [13] and COI/T.20/Doc. No. 22, 2005 [14]. The expert panel was composed of 10 assessors. A specific profile sheet was prepared including the following attributes: green or ripely fruit, sweet, bitter, pungent, grass, artichoke, herbs, tomato, almond. Since it was the first time that the group of tasters scored such a great number of different monocultivars oils we decided to add two lines for "others" attributes and it was specified that the tasters could use all the possible retronasal, aromatic olfactory sensations described in COI/T.20/Doc. No. 22, 2005 but the tasters were also advised to describe any other possible personal recognized sensation. Only when the majority of tasters scored "others" the value of the intensity of the described sensation was used for the statistics performed over the oils. Medians of the attributes were calculated as in COI/T.20/Doc. No. 15/Rev. 1, 1996. Basic statistics and UPGMA cluster analysis were performed by the Systat 11 program (Systat Software Inc. Richmond, CA, USA).

\section{RESULTS}

The list of the 57 cultivars from which it was possible to harvest the quantity of fruit necessary for the production of the oil is showed in Table $\mathbf{1}$, they represent $72 \%$ of the total number of cultivars in collection.

The names of the cultivars are listed following the order of the harvesting and each date is presented also as day of the year (DOY). The harvesting period was of 48 days, starting with Melaiolo at the middle of October and ending with Leccio del Corno at the beginning of December. The date of the harvesting and the stage of ripening of the fruits affects the chemical characteristics of the produced oils and for this reason we tried to pick the fruits in a reduced range of the maturity index. About $67 \%$ of the cultivars were harvested with values of the index between 2 and 3,9 cultivars were picked with fruits still yellowish green $(M I<2)$ and 10 with reddish or black skin (MI>3). These values of MI are considered optimal for the quality of the oil that can be produced. All the oils produced along the season presented values of acidity below $0.8 \%$ and peroxides $<20$ milliequivalents of active oxygen $/ \mathrm{kg}$ oil (data not showed) and could be classified as extra virgins.

The mean values of the intensity of perception of each attribute evaluated by the panel of experts are reported in Table $\mathbf{2}$ where for each variable are also showed the minimum and maximum values among the samples, standard deviation, coefficient of variation and number of oil samples in which each single attributes was scored.

In seven oils the panel found other attributes listed in Table 3. In some of the oils like that one of Americano, Cucca, Leccio Maremmano and Tisignana were scored two or three different attributes while in Cuoricino, Gremigna Tonda and Lastrino the majority of the tasters scored a single sensation of respectively sweet pepper, camomile, and banana.

Using the value of each organoleptic attribute as parameters for the calculation of the dissimilarity among the oils it was possible to cluster the 57 monocultivar oils and the plot 
Table 1. Code and Name of the Cultivars Belonging to the Tuscan Germplasm Used for the Production of the 57 Monocultivar Extra Virgin Olive Oils. The Cultivars are Listed in the Order of the Harvesting Date Expressed Also as Day of the Year (DOY)

\begin{tabular}{|c|c|c|c|c|c|c|c|}
\hline Code & Name & Date & DOY & Code & Name & Date & DOY \\
\hline 1 & Melaiolo & $15 / 10$ & 288 & 30 & Mignolo & $5 / 11$ & 309 \\
\hline 2 & Maurino & $15 / 10$ & 288 & 31 & Arancino & $5 / 11$ & 309 \\
\hline 3 & Olivo S. Lorenzo & $18 / 10$ & 291 & 32 & Morchiaio & $5 / 11$ & 309 \\
\hline 4 & Leccino & $18 / 10$ & 291 & 33 & Leccione & $5 / 11$ & 309 \\
\hline 5 & Scarlinese & $19 / 10$ & 292 & 34 & Rossellino Cerretano & $5 / 11$ & 309 \\
\hline 6 & Pendolino & $19 / 10$ & 292 & 35 & Aretino & $11 / 11$ & 315 \\
\hline 7 & Ginestrino & $19 / 10$ & 292 & 36 & Lazzero Pratigiano & $19 / 11$ & 323 \\
\hline 8 & Filare & $20 / 10$ & 293 & 37 & Rossello & $20 / 11$ & 324 \\
\hline 9 & Madonna impruneta & $20 / 10$ & 293 & 38 & Lastrino & $20 / 11$ & 324 \\
\hline 10 & Colombana & $20 / 10$ & 293 & 39 & Moraiolo & $20 / 11$ & 324 \\
\hline 11 & Colombino & $20 / 10$ & 293 & 40 & Gremigna Tonda & $23 / 11$ & 327 \\
\hline 12 & Cuoricino & $21 / 10$ & 294 & 41 & Punteruolo & $23 / 11$ & 327 \\
\hline 13 & Rossino & $21 / 10$ & 294 & 42 & Quercetano & $24 / 11$ & 328 \\
\hline 14 & Gremigno di Fauglia & $21 / 10$ & 294 & 43 & Lazzero & $24 / 11$ & 328 \\
\hline 15 & Emilia & $22 / 10$ & 295 & 44 & Mortellino & $24 / 11$ & 328 \\
\hline 16 & Lazzero di Prata & $22 / 10$ & 295 & 45 & Americano & $25 / 11$ & 329 \\
\hline 17 & Allora & $22 / 10$ & 295 & 46 & Da Cuccare & $26 / 11$ & 330 \\
\hline 18 & Olivastra Seggianese & $22 / 10$ & 295 & 47 & Gremignolo di Bolgheri & $26 / 11$ & 330 \\
\hline 19 & Pendagliolo & $26 / 10$ & 299 & 48 & Correggiolo & $27 / 11$ & 331 \\
\hline 20 & Giogolino & $26 / 10$ & 299 & 49 & Cucca & $27 / 11$ & 331 \\
\hline 21 & Madremignola & $26 / 10$ & 299 & 50 & Olivo del Mulino & $29 / 11$ & 333 \\
\hline 22 & Lazzero delle Guadalupe & $27 / 10$ & 300 & 51 & Frantoio & $29 / 11$ & 333 \\
\hline 23 & Tisignana & $27 / 10$ & 300 & 52 & Ciliegino & $30 / 11$ & 334 \\
\hline 24 & Rosino & $28 / 10$ & 301 & 53 & Mignolo Cerretano & $30 / 11$ & 334 \\
\hline 25 & Pesciatino & $28 / 10$ & 301 & 54 & Olivo di Casavecchia & $1 / 12$ & 335 \\
\hline 26 & Mansino & $29 / 10$ & 302 & 55 & Puntino & $1 / 12$ & 335 \\
\hline 27 & Tondello & $29 / 10$ & 302 & 56 & Leccio Maremmano & $2 / 12$ & 336 \\
\hline 28 & Piangente & $4 / 11$ & 308 & 57 & Leccio del Corno & $2 / 12$ & 336 \\
\hline 29 & S. Francesco & $4 / 11$ & 308 & & & & \\
\hline
\end{tabular}

Table 2. Minimum, Maximum, Mean, Standard Deviation and Coefficient of Variation of Each Descriptor Within the 57 Monocultivar EVOOs. The Number of Samples Indicates the Number of Oils with the Correspondent Descriptor. SD=Standard Deviation $\mathrm{CV}=$ Coefficient of Variation

\begin{tabular}{|c|c|c|c|c|c|c|}
\hline Descriptor & Min & Max & Mean & SD & $\mathrm{CV}$ & No of Samples \\
\hline Greenly fruit & 2.8 & 6.7 & 5.2 & 0.1 & 0.13 & 54 \\
\hline Ripely fruit & 0 & 3.3 & 0.6 & 0.9 & 1.49 & 22 \\
\hline Sweet & 0 & 2.9 & 1.1 & 0.9 & 0.76 & 40 \\
\hline Bitter & 3.5 & 6.0 & 4.6 & 0.5 & 0.10 & 57 \\
\hline Pungent & 3.1 & 6.0 & 4.9 & 0.5 & 0.10 & 57 \\
\hline Grass & 0 & 5.0 & 2.6 & 0.7 & 0.29 & 56 \\
\hline Artichoke & 0 & 3.0 & 2.2 & 0.7 & 0.32 & 55 \\
\hline Herbs & 0 & 2.2 & 0.2 & 0.5 & 2.38 & 11 \\
\hline
\end{tabular}


Table 2. cont....

\begin{tabular}{|c|c|c|c|c|c|c|}
\hline Descriptor & Min & Max & Mean & SD & CV & No of Samples \\
\hline \hline Tomato & 0 & 2.8 & 0.2 & 0.6 & 2.5 & 10 \\
\hline Almond & 0 & 2.0 & 0.1 & 0.4 & 3.9 & 4 \\
\hline Others & 0 & 1.7 & 0.1 & 0.3 & 3.5 & 7 \\
\hline
\end{tabular}

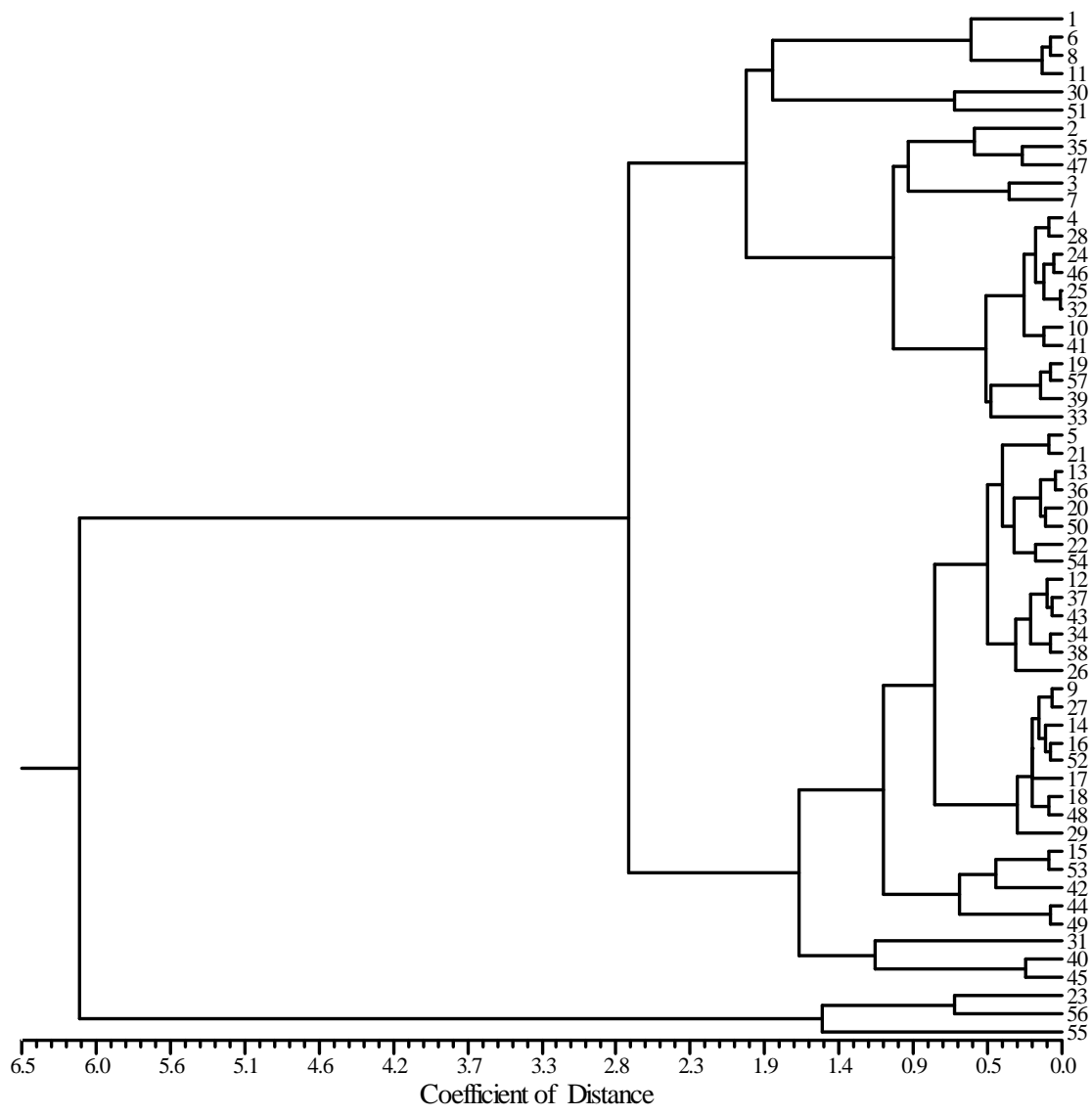

Fig. (1). Plot of tree produced by UPGMA cluster analysis of the 57 Tuscan monocultivar extra virgin olive oils using the value of each organoleptic attribute as parameter for the calculation of the dissimilarity among oils. Using a distance value of 2.7 it is possible to evidence three different clusters. The features of the oils of each cluster are reported in Table 4. The numbers correspond to the code of the cultivar listed in Table 1.

of the tree produced by UPGMA cluster analysis is showed in Fig. (1).

Table 3. Olfactory Sensations Described by the Tasters within the 7 Oils with Others Descriptors as Indicated in Table 3.

\begin{tabular}{|c|c|c|}
\hline Code & Cultivar & Olfactory sensations \\
\hline \hline 45 & Americano & Flowers, mature fruits (melon, apple) \\
\hline 49 & Cucca & Mature fruits (melon), mushroom \\
\hline 12 & Cuoricino & Sweet pepper \\
\hline 40 & Gremigna Tonda & Camomile \\
\hline 38 & Lastrino & Exotic fruit (banana) \\
\hline 56 & Leccio Maremmano & Flowers, mushroom \\
\hline 23 & Tisignana & Flowers, mature fruits \\
\hline
\end{tabular}

The mean values of each attribute within each of the three clusters of oils are showed in Table 4 . In the same table are also showed the values expressed by the evaluators relatively to the color of the oils, the mean date of harvesting and the total biophenols content averaged among the oils belonging to the group.

\section{DISCUSSION}

As it can be seen in Table $\mathbf{3}$ some attributes like bitter and pungent were found by the tasters in all the samples since these two sensations are recognized as the main components of the freshly produced EVOO. The intensity of both of them had a similar range varying from about 3-3.5 to 6 . Bitter and pungent are, of course, correlated each other $(r=0.60)$ and to the presence of other unripe attributes like grass or artichoke that were both described in almost all the oils. This strong presence of unripe sensations in the oils was due to the low MI of the fruits used for the oil production which caused 54 oils over 57 to be recognized from the 
Table 4. Clusters, Number of Oil Samples in Each Cluster and Mean Value ( \pm Standard Error of the Mean) of Each Organoleptic Attribute within the Cluster. For each cluster is also Indicated the Mean Value of the Color Attribute, Date of Harvesting (doy) Maturity Index (mi) and Biophenol Content (mg/kg)

\begin{tabular}{|c|c|c|c|}
\hline Variable & Cluster 1 & Cluster 2 & Cluster 3 \\
\hline & $\mathbf{2 3}$ & $5.0 \pm 0.1$ & $4.3 \pm 0.3$ \\
\hline Greenly fruity & $5.6 \pm 0.1$ & $0.7 \pm 0.1$ & $3.0 \pm 0.2$ \\
\hline Ripely fruit & $0.1 \pm 0.1$ & $1.5 \pm 0.1$ & $2.2 \pm 0.4$ \\
\hline Sweet & $0.4 \pm 0.1$ & $4.5 \pm 0.1$ & $4.0 \pm 0.3$ \\
\hline Bitter & $4.9 \pm 0.1$ & $4.9 \pm 0.1$ & $4.1 \pm 0.5$ \\
\hline Pungent & $5.2 \pm 0.1$ & $2.3 \pm 0.1$ & $1.1 \pm 0.6$ \\
\hline Grass & $3.0 \pm 0.1$ & $2.1 \pm 0.1$ & $0.4 \pm 0.4$ \\
\hline Artichoke & $2.5 \pm 0.1$ & $0.1 \pm 0.1$ & 0 \\
\hline Herbs & $0.3 \pm 0.1$ & 0 & 0 \\
\hline Tomato & $0.5 \pm 0.2$ & 0 & $0.9 \pm 0.6$ \\
\hline Almond & $0.2 \pm 0.1$ & 0 & $0.3 \pm 0.3$ \\
\hline Others & $0.1 \pm 0.1$ & $3.4 \pm 0.3$ & $0.6 \pm 0.3$ \\
\hline Green & $4.1 \pm 0.3$ & $4.8 \pm 0.2$ & $5.3 \pm 1.3$ \\
\hline Yellow & $4.3 \pm 0.3$ & $312 \pm 3$ & $309 \pm 12$ \\
\hline DOY & $307 \pm 3$ & $2.6 \pm 0.1$ & $3.2 \pm 0.1$ \\
\hline MI & $2.4 \pm 0.1$ & $450 \pm 27$ & $281 \pm 30$ \\
\hline Total biophenols & $570 \pm 37$ & & \\
\hline
\end{tabular}

tasters mainly as oils with greenly fruit overall sensation [15]. The presence of ripely fruit attributes in 22 samples was due to the fact that the tasters experienced a green/ripe mixed flavor in 19 oils while only 3 were completely ripely fruit, hence 32 samples had only completely greenly fruit sensation. The score of the ripely fruit sensation was always lower compared to that one of greenly fruit, reaching a maximum median of 3.3 within the samples. The presence of the sweet sensation was recorded in 40 oils but with low median (maximum 2.9). The attributes of herbs and tomato was found in a similar number of samples (about 18-19\% of the total) while the attribute of almond was found only in 4 oils. In 7 oils ( $12 \%$ of the total) was possible to score the "others" attributes listed in Table 4. Most of them like flowers, apple, camomile, banana, sweet pepper, are included in the list of the attributes listed in IOOC, while others like melon, always well described independently by the tasters, could be classified like as "exotic fruit" sensation together with banana. The presence of the mushroom sensation in two different samples could be due to the presence of a defect in the product. Some of the fruits of these two cultivars were partially affected by the attack of a fungus (Colletotrichum gloesporioides Penzig). The mould evidently was not enough to ruin completely the quality of the oils, since they had all the chemical characteristics to be classified as extra virgins, but could be detected as a peculiar sensation by the tasters. Saffron, chestnut flour and coffee were the organoleptic attributes found from few tasters in some of the oils. Since the oils were produced at same condition we cannot consider these as defects, due for example to the presence of high temperature during malaxation or mechanical action of the crusher on the stones. The numbers of assessors finding these attributes was always too low to assure the security that that peculiar sensation was distinctive of that particular oil and we did not consider the score on statistics of Table $\mathbf{3}$ but always more than one taster ( 2 to 4 ) found the same character independently. This could be a signal of a drawback of the oil organoleptic assessment: the panel is used to evaluate hundreds of oils to determine if they contain any defects, hence cannot be considered extra virgin or virgin or, if operating within a GPI or PDO they can be able to tell if the samples own the characteristics of the determined origin, in our case the Tuscan PGI for instance, require mostly artichoke and grass, but the description of a novel sensation, not included in the list of the descriptors, can become difficult. Although we tried to harvest the fruit at the same MI we had a variation of this index within the samples and his known that the ripening influences the phenols content and the organoleptic features of the EVOO [16]. We found, within each of the three clusters, oils with more homogenous ripening stage of the fruits. In the cluster number three the statistics included 3 oils with higher MI (3.2) compared to those in the cluster two $(\mathrm{MI}=2.6)$ or one $(\mathrm{MI}=2.4)$. Also the total phenols of the oils of this small group was lower since it is well known that during the ripening there is a decrease of the total phenols in the fruits and in the produced oils $[15,17]$. Although is not an organoleptic attribute considered in the official COI test, the color can be highly considered during an hedonistic evaluation of an oil sample and as it can be seen in Table 4 the oils in cluster three were more yellow and less green compared to all the others. The oils belonging to cluster 3 had also the presence of sensation of almond, flowers and soft fruits. The use of more ripe fruits could not be considered only negatively because evidently could produce oils less aggressive on the palate, with positive sensations of sweetness and pleasant flavor of mature fruits and flowers all characteristics that can positively affect the food acceptability by the consumers. Of course their lower phenols content could not protect them for a long time [18] during the storage and they will probably lose their positive at- 
tributes in a shorter time compared to other oils with higher phenols content $[19,20]$. In the cluster number two 31 samples are grouped, representing the largest percentage of oils within the collection (54\%). They had an average phenols content of $450 \mathrm{mg} / \mathrm{kg}$, positive green attributes (Greenly fruit, grass, artichoke) associated to the presence of ripely fruit sensations (sweet) but with low herbs sensation and any tomato or "others". The oils grouped in the cluster number one were more green than the others, with very low ripe fruity sensations, with presence of tomato and herbs attribute. Also the presence of "others" olfactive attributes was scored, mainly due to green olfactory sensations of olive leaf, sweet pepper and fig leaf. The values of the bitter and pungent attributes are the higher among the others due to the highest phenols content, not depending on MI and DOY since these did not differ from those of cluster number two, but to the genetic origin of the oils. The genetic pool is also important in olive for the acidic profile of the oils: we did not showed here the lipid composition of the samples analysed and preliminarily exposed [21] but it is important to underline that also the composition of the lipid matrix can plays a key role in the perception of some attributes [22] in virgin olive oil, since for instance polyunsaturated matrices produce milder sensations and are less bitter than monounsaturated ones [23]. The samples belonging to the cluster number three had an average content of polyunsaturated acids of $17 \%$, more than the double, than those of cluster two $(7.3 \%)$ or one $(7.4 \%)$. This suggests that the knowledge about the organoleptic profile of the oil that can be obtained by each variety must be taken into consideration, keeping stable the extraction variables, maturity index and genetically constitutional features as phenols and lipidic matrix.

\section{CONCLUSIONS}

With this work we showed that the sensory profile of the extra virgin olive oil produced from a single variety is deeply affected primarily by the maturity index of the fruits and correlated to both the phenols content and acidic profile, but these two variables depend on the genetic matrix hence the cultivar plays a fundamental role on the overall quality. Within the studied set only a small percentage of oils held peculiar and distinctive organoleptic attributes. At a practical level the work evidenced several varieties that for the intensity of organoleptic sensations, equilibrium among attributes or peculiar features could represent a step forward respect to the average quality level of the production realized using the widest cultivated varieties of the region. The majority of the varieties can produce oils with the attributes requested by the local PGI or PDO while others could give sensations presently not considered as tomato or herbs. The presence of peculiar easily assessable organoleptic attributes, like tomato, sweet pepper, camomile or others is not common within the olive oils and they are present only to few varieties within the Tuscan germplasm collection. The list of possible positive descriptors could be enlarged or better specified including the terms of melon and chestnut flour. We proposed also to take into consideration the olfactory sensation of mushroom as reminiscent of the typical and pleasant odour of fresh cut mushroom or fresh "porcini" mushroom like a possible new negative attribute of the extra virgin olive oil induced by the presence of antrachnose (Colletotrichum gloesporioides; Glomerella cingulata) on the olive fruits.

\section{CONFLICT OF INTEREST}

The author confirms that this article content has no conflicts of interest.

\section{AKNOWLEDGEMENTS}

We are grateful to Alessandra Betti and Andrea Caturelli for their valuable help during the harvesting, milling and preparation of the oil samples.

\section{REFERENCES}

[1] Alfei B, Magli M, Rotondi A, Pannelli G. Statistical analyses of sensory properties of Italian monovarietal olive oils. Proceedings II International Seminary "Biotechnology and Quality of Olive tree Products around the Mediterranean basin"; 2006 Nov 5-10; del Vallo Marsale: Mazara (TP) 2006; vol. 5-10: pp. 431-4.

[2] Morales MT, Tsimidou M. The role of volatile compounds and polyphenols in olive oil sensory quality. In: Harwood J, Aparicio R. Eds. Handbook of olive oil. Analysis and Properties. Gaithersburg, MD (USA): Aspen Publication 2000; pp. 393-58.

[3] Angerosa, F, Mostallino R, Basti C, Vito R. Virgin olive oil odour notes: their relationships with volatile compounds from the lipoxygenase pathway and secoiridoid compounds. Food Chem 2000; 68: 283-7.

[4] Angerosa F, Servili M, Selvaggini R, Taticchi A, Esposto S, Montedoro G. Volatile compounds in virgin olive oil: Occurrence and their relationship with the quality. J Chromatogr 2004; 1054: 1731.

[5] Procida G, Giomo A, Cichelli A, Conte LS. Study of volatile compounds of defective virgin olive oils and sensory evaluation: a chemometric approach. J Sci Food Agric 2005; 85 (13): 2175-83.

[6] Angerosa A. Influence of volatiles compounds on virgin olive oil quality evaluated by analytical approaches and sensor panels. Eur J Lipid Sci Technol 2002; 104: 639-60.

[7] Ranalli A, Modesti G, Patumi M, Fontanazza G. The compositional quality and sensory properties of virgin olive oil from a new olive cultivar - I77. Food Chem 2000; 69(1): 37-46.

[8] Luna G, Morales MT, Aparicio R. Characterisation of 39 varietal virgin olive oils by their volatile compositions Food Chem 2006; 98(2): 243-52.

[9] Caporale G, Policastro S, Carlucci A, Monteleone E. Consumer expectations for sensory properties in virgin olive oils. Food Qual Prefer 2006; 17: 116-25.

[10] Cantini C, Cimato A, Sani G. Morphological evaluation of olive germplasm present in Tuscany region. Euphytica 1999; 109: 173-81.

[11] Cantini C, Cimato A, Autino A, Redi A, Cresti M. Assessment of the tuscan olive germplasm by micro satellite markers reveal genetic identities and different discrimination capacity among and within cultivars. J Am Soc Hortic Sci 2008; 133(4): 1-7.

[12] Uceda M, Frías L. Épocas de recolección. Evolución del contenido graso del fruto y de la composición y calidad del aceite, In: IOOC Ed. Proceedings of II Seminario Oleícola International. Córdoba: Spain, 1975.

[13] International Olive Oil Council (IOOC). 1996. Sensory analysis of olive oil. Organoleptic assessment of virgin olive oil. COI/T.20/Doc.No.15/Rev.1. 1996. Available at http://www.international-oliveoil.org/estaticos/view/224-testing-methods

[14] International Olive Oil Council (IOOC). Method for the organoleptic assessment of extra virgin olive oil applying to use a designation of origin COI/T.20/Doc. n 22. 2005. Available at http:// www.internationaloliveoil.org/estaticos/view/224-testing-methods

[15] Aparicio R, Morales MT. Characterization of olive ripeness by green aroma compounds of virgin olive oil. J Agric Food Chem 1998; 46: 1116-22.

[16] Tsimidou M. Polyphenols and quality of virgin olive oil in retrospective. Ital J Food Sci 1998; 10: 99-15.

[17] Dag A, Kerem Z, Yogev N, Zipori I, Lavee S, Ben-David E. Influence of time of harvest and maturity index on olive oil yield and quality. Sci Hortic 2011; 127: 358-66.

[18] Baldioli M, Servili M, Peretti G, Montedoro GF. Antioxidant activity of tocopherols and phenolic compounds of virgin olive oil. J Am Oil Chem Soc 1996; 73: 1589-93.

[19] Romani A, Lapucci C, Cantini C, Ieri F, Mulinacci N, Visioli F. Evolution of minor polar compounds and antioxidant capacity dur- 
ing storage of bottled extra virgin olive oil. J Agric Food Chem 2007; 55: 1315-20.

[20] Gutierrez F, Fernandez J. Determination parameters and components in the storage of virgin olive oil. Prediction of storage time beyond which the oil is no longer of "extra" quality. J Agric Food Chem 2002; 50: 571-7.

[21] Cantini C, Sani G, Betti A, Migliorini M, Mazzanti L. Fatty acid and phenolic composition of monocultivar oils from the Tuscan olive germplasm: health quality assessment and classification. $4^{\text {th }}$ International Conference "Oliviculture, Biotechnology and Quality of Olive Tree Products" (OLIVEBIOTEQ 2011); 2011 Oct $31^{\text {st }}$ Nov $4^{\text {th }}$; Chania, Crete: Greece 2011.

[22] Koriyama T, Wongso S, Watanabe K, Abe H. Fatty acid composition of oil species affects the 5 basic taste perceptions. J Food Sci 2002; 67: 868-73.

[23] García-Mesa JA, Pereira-Caro G, Fernández-Hernández A, GarcíaOrtíz Civantos C, Mateos R. Influence of lipid matrix in the bitterness perception of virgin olive oil. Food Qual Prefer 2008; (4): 421-30.

(c) Cantini et al.; Licensee Bentham Open.

This is an open access article licensed under the terms of the Creative Commons Attribution Non-Commercial License (http://creativecommons.org/licenses/by-nc/3.0/) which permits unrestricted, non-commercial use, distribution and reproduction in any medium, provided the work is properly cited. 